\title{
The Design and Construction of the HELIX RICH Detector
}

\author{
P. Allison ${ }^{1}$, J. J. Beatty ${ }^{1}$, L. Beaufore ${ }^{2}$, Y. Chen ${ }^{3}$, S. Coutu ${ }^{3}$, E. Ellingwood ${ }^{4}$, \\ M. Gebhard ${ }^{5}$, N. Green ${ }^{6}$, D. Hanna ${ }^{4}$, B. Kunkler ${ }^{5}$, I. Mognet ${ }^{3}$, R. Mbarek ${ }^{2}$, \\ K. McBride ${ }^{1}$, K. Michaels ${ }^{5}$, D. Müller ${ }^{2}$, J. Musser ${ }^{5}$, S. Nutter ${ }^{7}$, S. O’Brien ${ }^{4}$, N. Park ${ }^{8}$, \\ T. Rosin ${ }^{4}$, E. Schreyer ${ }^{2}$, G. Tarlé ${ }^{6}$, M. Tabata ${ }^{9}$, A. Tomasch ${ }^{6}$, G. Visser $^{5}$, \\ S. P. Wakely ${ }^{2}$, T. Werner ${ }^{2}$, I. Wisher ${ }^{* 2}$, and $\mathbf{M}$. $\mathbf{Y u}^{3}$ \\ ${ }^{1}$ The Ohio State University, Columbus, OH 43210 USA \\ ${ }^{2}$ University of Chicago, Chicago, IL 60637 USA \\ ${ }^{3}$ Pennsylvania State University, University Park, PA 16802 USA \\ ${ }^{4}$ McGill University, Montreal, QC H3A 2T8, Canada \\ ${ }^{5}$ Indiana University, Bloomington, IN 47405 USA \\ ${ }^{6}$ University of Michigan, Ann Arbor, MI 48109 USA \\ ${ }^{7}$ Northern Kentucky University, Highland Heights, KY 41099 USA \\ ${ }^{8}$ University of Wisconsin-Madison, Madison, WI 53706 USA \\ ${ }^{9}$ Chiba University, Chiba, 263-8522, Japan
}

\begin{abstract}
The High-Energy Light Isotope eXperiment (HELIX), is a new balloon-borne 1 Tesla magnet spectrometer to measure light isotopic abundances including ${ }^{10} \mathrm{Be}$ and ${ }^{9} \mathrm{Be}$ to energies of $\sim 3$ $\mathrm{GeV} /$ nuc. This requires precise measurements of the rigidity and velocity of the detected particles, necessitating a multi-system detector. The detectors include a magnet/particle tracker for measuring the rigidity, and both time-of-flight and ring imaging Cherenkov (RICH) detectors for measuring the particle velocity and charge. The energy range of interest, high altitude environment, and proximity to the magnet present a challenging environment for a RICH detector. HELIX uses a proximity-focused RICH with an aerogel radiator above a $1 \mathrm{~m}^{2}$ focal plane populated with 12,800 silicon photomultipliers (SiPMs). In this contribution we will describe the design of the RICH and discuss progress towards the fabrication and characterization of the focal plane components.
\end{abstract}

36th International Cosmic Ray Conference -ICRC2019-

July 24th - August 1st, 2019

Madison, WI, U.S.A.

\footnotetext{
*Speaker.

${ }^{\dagger}$ E-mail:wisher@uchicago.edu
} 


\section{Introduction}

The High Energy Light Isotope eXperiment (HELIX) is a balloon-borne detector designed to accurately measure light cosmic-ray isotopes from $\sim 0.2 \mathrm{GeV} / \mathrm{n}$ to above $\sim 3 \mathrm{GeV} / \mathrm{n}[1,2]$. HELIX is a magnet spectrometer which consists of a 1 Tesla magnet, a gas tracker system, time-of-flight counters (TOF), and a ring-imaging Cherenkov (RICH) detector. It is currently planned to fly out of McMurdo Station in Antarctica during the austral summer of 2020/2021. Though HELIX will observe a wide range of light isotopes, the primary goal is a measurement of the ratio of ${ }^{10} \mathrm{Be}$ and ${ }^{9} \mathrm{Be}$. Both isotopes are created via spallation of heavier nuclei but ${ }^{10} \mathrm{Be}$ is unstable, decaying with a half life of $1.4 \times 10^{6} \mathrm{yrs}$. The ratio of the two isotopes of beryllium can then serve as an isotopic clock to determine the average propagation time of the cosmic rays through the galaxy. Though the beryllium ratio has been measured previously to energies of $\sim 1.5 \mathrm{GeV} / \mathrm{n}$ [3], a more precise measurement to higher energies is needed to better constrain propagation models [4].

A key requirement for mass reconstruction in magnet spectrometers is the accurate reconstruction of the velocity of the through-going particles. HELIX uses a RICH detector to perform these measurements, due to their superior performance at high energy, vs, e.g., time-of-flight or integrating Cherenkov counters. In this proceeding, we will present an overview of the HELIX RICH, describe some technical challenges, and give a status of the detector development. A description of the rest of the detector systems can be found in these proceedings [5].

\section{RICH Design Overview}

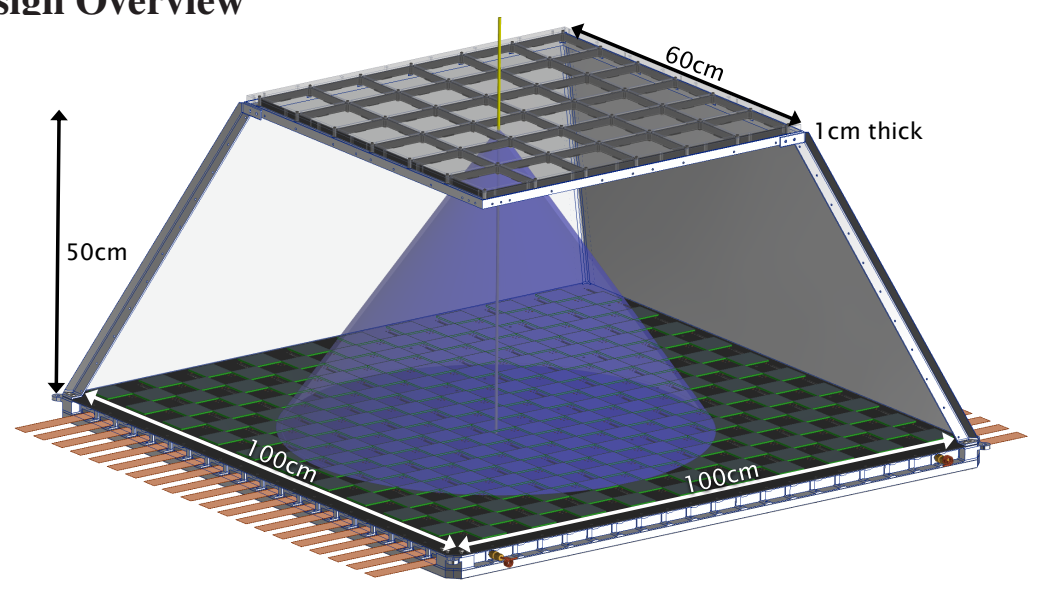

Figure 1: Schematic of the HELIX RICH showing an example particle in yellow with a Cherenkov cone in blue. The dimensions of the aerogel radiator assembly, the expansion length and the size of the detector plane are shown.

RICH detectors utilize the velocity dependence of the emission angle of Cherenkov radiation, to accurately measure relativistic charged particle velocities. The main components of a RICH detector are a radiator assembly to produce the Cherenkov light and a detector plane to image the resulting ring. Though all RICH detectors measure the angular dependence, they can be designed with a variety of different geometries, materials, and photon detectors depending on the spatial requirements and the energies of interest. Due to the space requirements of the balloon payload, the geometry of the HELIX RICH is proximity focused as seen in Figure 1. This is the natural choice because the design remains compact and it requires no focusing optics, which would complicate the assembly and characterization. 
The HELIX radiator consists of an aerogel plane made up of $10 \mathrm{~cm}$ by $10 \mathrm{~cm}$ by $1 \mathrm{~cm}$ thick tiles with a nominal index of refraction of $n=1.16$. This index of refraction is the highest refractive index ever used as Cherenkov imaging radiators [6] and provides a low threshold velocity of $\sim$ $0.87 c$. This allows the RICH energy range to overlap with the upper energy range of the time of flight system and provide a way to cross check the velocity resolution of both systems. The tiles were manufactured by M. Tabata at Chiba University with the final set of tiles finished in 2018 [7] and are currently being characterized using multiple methods [8].

The aerogel thickness and index of refraction set the pixel size of the SIPMs and thus inform the expansion length. The pixel size should be less than the thickness of the imaged ring and the expansion length large enough to allow the radius to move across pixels with changing velocity. The RICH detector plane will be located $50 \mathrm{~cm}$ from the aerogel and cover $1 \mathrm{~m}^{2}$ with $6 \mathrm{~mm} \times 6 \mathrm{~mm}$ Silicon Photomultiplier (SiPM) pixels aranged in $8 \times 8$ modules of 64 pixels. These modules are organized in a checkerboard pattern, yielding a total of 200 SiPM arrays or 12800 pixels in the RICH focal plane. The use of SiPMs is novel for a balloon-borne RICH and leads to several constraints on the design of the assembly and electronics. The characterization of different SiPM options and final selection process is discussed in the following section.

\subsection{Detector Technology Selection}

Traditionally, when choosing the optical detectors for the RICH detector plane, multianode Photomultiplier Tubes (PMTs) are selected. PMTs have the advantage of exhibiting relatively low dark rates (on the order of $\mathrm{kHz}$ ), favorable pixel sizes, high gain, good dynamic range, and relative temperature insensitivity. Despite these advantages, the proximity of the RICH focal plane to the HELIX magnet is problematic. Stray magnetic fields on the order of hundreds of Gauss will negatively impact the gain and detection efficiency of a PMT, reducing the expected signal from emitting particles. Furthermore, due to the relatively high value of the field and the total volume of detectors to be protected, magnetic shielding would require an impractical amount of material for a balloon payload. Silicon photomultipliers, which are insensitive to magnetic fields, are hence chosen for the HELIX RICH. These have the added benefit of requiring relatively small bias voltages, which avoids the added complications of high-voltage power distribution at float.

\subsection{SiPM Behavior and Characterization}

SiPMs consist of multiple avalanche photodiodes reversed-biased with series quenching resistors, all wired in parallel. SiPMs have their own set of properties that must be measured and accounted for in the design. The properties of interest when evaluating SiPMs for a RICH detector are: the photon detector efficiency (PDE), the gain, the dark count rate, the optical cross talk, and individual avalanche diode pixel size.

Ideally, to maximize the number of detected photons, the PDE should be as large as possible. The gain should be high enough for compatibility with the data acquisition electronics, and there should be good separation between electronics noise and the single photoelectron (PE) signal amplitude. The dark rate is required to be as low as possible to reduce the number of spurious hits that contribute noise to the detected Cherenkov ring and to limit signal pile-up that will degrade the charge resolution.

The optical cross talk is a unique property of SiPMs, where the avalanche produced by a single photon produces an additional detectable photon in the pixel. This causes charge promotion of hits 
or a single photon to be observed as 2 or more photons. The cross talk does not strongly affect the geometry of the Cherenkov ring except that it makes some cleaning algorithms less effective.

The individual avalanche photodiode cell size and the size of the device cells determine the number of photodiodes per pixel, the fill factor, and the saturation level of the device. When choosing the cell size, there is a trade off between number of cells which determines the light level where the device will saturate and the fill factor which affects detector efficiency. With more but smaller cells, the increased number of cell-to-cell gaps, which are insensitive to photons, become more significant and the efficiency of the device will suffer. Due to the relatively low per-pixel photon counts of the HELIX RICH detector, saturation effects are negligible, with at most dozens of photons expected in a single pixel. In this case, a large cell size of $75 \mu \mathrm{m}$ is chosen to maximize the detection efficiency of the SiPM and the signal in the RICH.

Several samples were analyzed from commercial and academic sources in order to determine the optimal SiPM technology choice for the RICH instrument, with a total of 14 different models being tested. The samples were characterized using a test stand at University of Chicago, designed to evaluate the devices with varying operating points, light intensities, and temperatures ranging from $-10^{\circ} \mathrm{C}$ to $40^{\circ} \mathrm{C}$.

\subsection{RICH SiPM Part Selection}

Measurements of the gain, cross talk, dark rate, and relative quantum efficiency were made for each sample. The measured values were then used to model noise levels in a simulation of physics events to determine the impact on the RICH resolution. SiPMs can be either coated with silicone, acrylic, or have no protective coating over the pixels. Different coatings affect what wavelengths the SiPMs are sensitive to and the optical cross talk rate.

After analyzing models with different

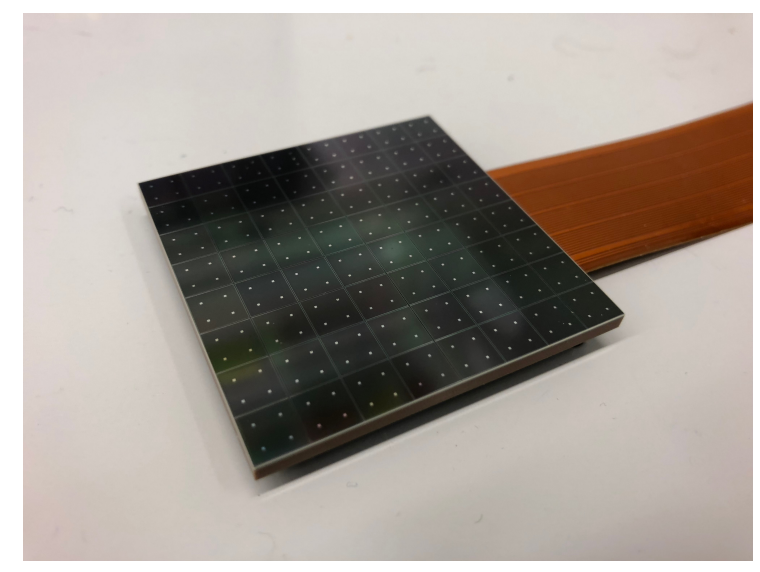

Figure 2: Photo of the $8 \times 8 \mathrm{SiPM}$ array with the attached $70 \mathrm{~cm}$ flexible printed circuit cable. values of dark rate, cross talk, and PDE, the most important parameter for the $\beta$ resolution of the RICH was determined to be the dark rate of the pixels. The cross talk merely promotes hits to higher charge which effects the charge resolution of the RICH but has little affect on the location of the Cherenkov Ring. The PDE of the different pixels did not vary enough to affect the selection of the pixel technology. The dark rate being the dominant contributor to the resolution, the Hamamatsu LVR series was chosen, due to a lower dark rate compared to other samples.

Unfortunately, the dark rate of the SiPMs is affected by the impurities in the wafer and is the quantity that is hardest to control for in the manufacturing. To account for the variability in dark rate between manufactured pixels the RICH plane is still required to be temperature controlled to mitigate the effect of pixels that are manufactured with higher dark count rates.

The HELIX SiPM arrays were coated with a uniform layer of silicone over the pixels instead of an acrylic coating or leaving the pixels bare. The coating protects the pixels from environmental damage and simplifies the required handling procedures and has limited drawbacks for the RICH 
analysis. The silicone coating increases the optical cross talk rate of the SiPM. This additional cross talk effect is caused when photons created in the pixel during an avalanche reflect off the surface of the coating and back into a pixel causing a subsequent avalanche. In the SiPM samples analyzed, the coating doubled the cross talk rate of a non-coated pixel with silicone being slightly lower than acrylic. The coating also has the effect of attenuating photons especially in the UV end of the SIPMs wavelength response. While this does lower the number of detected photons it also limits the effect of any chromatic aberration that the radiator introduces. These competing effects limit the resolution gains one gets from removing the coating and make the silicone coating a more attractive option due to its other benefits.

To summarize, the HELIX RICH utilizes SiPMs with $75 \mu \mathrm{m}$ cells and $6 \times 6 \mathrm{~mm}^{2}$ pixels, which have been selected for low dark rate. The individual SiPMs are mounted onto a PCB in $8 \times 8$ arrays and coated with silicone to form a $50 \times 50 \mathrm{~mm}^{2}$ module. The module is a custom design, shown in Figure 2, which includes twin LMT70 temperature sensors, a high-density FX11B-110S-SV Hirose connector with an optimized pinout, and mounting posts. As of early 2019, the 206 SiPM arrays manufactured by Hamamatsu have been delivered and are undergoing full characterization to be integrated with the electronics and into the RICH.

\section{Readout System}

The RICH readout electronics have the challenging task of instrumenting a total of 12,800 SiPM channels with single PE charge resolution. There is an additional challenge due to the relatively low power availability on a balloon payload, requiring the electronics to limit their power per channel.

The HELIX RICH boards, seen in Figure 3, each read 8 SiPM arrays with 16 CITIROC1A chips each, requiring 25 boards total to instrument the focal plane. The CITIROC1A was chosen because it could meet the required readout specifications with minimum power consumption. The CITIROC1A from Weeroc is designed specifically for reading out multiple SiPM channels with 100ps timing and single PE charge resolution. Each chip can read out 32 SiPM pixels and provides fast trigger outputs for each channel as well as adjustable gain and bias voltage for tuning the operating points. Charge readout is accomplished via pulse height sampling with a variable time constant shaping circuit. The pulse heights are sampled with either a peak-sensing circuit or a sample-and-hold circuit.

The trigger produced by the ToF system will have $<10 \mathrm{~ns}$ jitter and the arrival time of photons on the RICH focal plane is narrow enough, that the sample-and-hold method is sufficient for charge sampling. This has the additional advantage over the peak-sensing circuit that requires less power per channel. Once sampled the pulse heights are read out via a shared multiplexed analog output to an ADC. Once read out the sampled data are packaged and sent back to the science flight computer over high speed serial links. This functionality has been tested and the boards have gone through prototyping and initial characterization of the readout performance with an example shown in Figure 3.

To keep the electronics out of the path of particles, custom designed $70 \mathrm{~cm}$ long flexible printed circuit board cables carry the SiPM signals from the focal plane to the edge where the electronics are located. The thin cables also further limit the amount of material as they have much less mass than an equivalent coaxial cable assembly. The cables were designed with a nominal impedance 

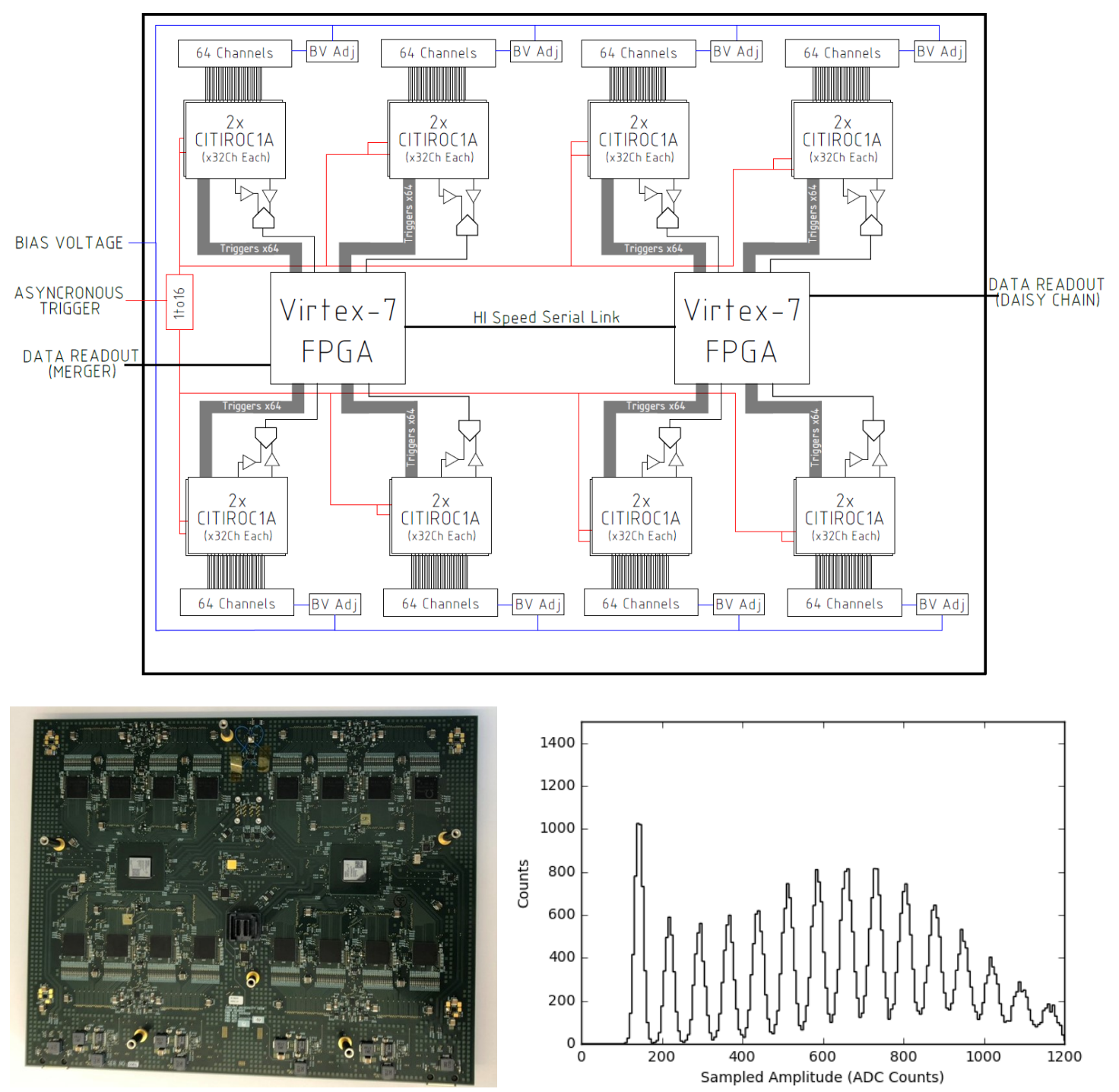

Figure 3: Top: Top level diagram of the RICH board showing a summary of the SiPM readout components. Bottom: Picture of the prototype RICH board without a heatsink to show the 2 FPGAs and 16 CITIROC1As for a total of 512 channel readout and a sample of a charge spectrum from a single SiPM using the full readout chain.

of $50 \mathrm{ohms}$ and the layers stacked up so the cathode voltage is supplied on the reference plane sandwiched between the two signal layers. This stack up forms the microstrip transmission lines and limits the inductance of the cable.

In 2019 the boards, cables, and downstream readout have gone through prototyping and initial characterization of the readout performance. With testing completed the full $25 \mathrm{RICH}$ boards and 200 cables will be manufactured in the Fall of 2019.

\section{RICH Mechanics}

The three main requirements of the RICH focal plane are that it remain flat to reduce aberrations, have low mass to reduce weight on the payload and material in the particle's path, and have 
a uniform cold temperature to minimize the impact of the temperature dependence of the SIPMs. Though there is not a cut off point at which a high temperature will cause the resolution to drop, a maximum operating point of $20^{\circ} \mathrm{C}$ was decided but a preferred operating point of $0^{\circ} \mathrm{C}$ was selected. To ensure that the SiPMs will remain at the desired temperatures, a thermal control system maintains the edges of the focal plane at a constant temperature. The power dissipation in the focal plane under normal conditions is under 1 Watt total due to the low dark current of less than 6 microamps for each SiPM. Under expected flight conditions, the dominant heating of the focal plane comes from other components, through conduction and radiation, rather than from the power dissipation in the SiPMs.

To minimize both the weight of the mechanical assembly and ensure that the focal plane is uniform, a $3 / 8^{\prime \prime}$ core honeycomb panel with thin 0.02 " facing sheets and aluminum closeouts was constructed. The closeouts are required to keep the assembly rigid against transverse forces on the honeycomb and to allow it to be supported easily from the 4 corners. To cost effectively meet the requirements, the facing sheets were laser cut and the closeouts machined separately and then the honeycomb plane assembled with the final dimensions of $42.5 " \times 42.5 " \times .55^{\prime \prime}$. To ensure the panels will survive the changes in pressure and temperature to be experi-

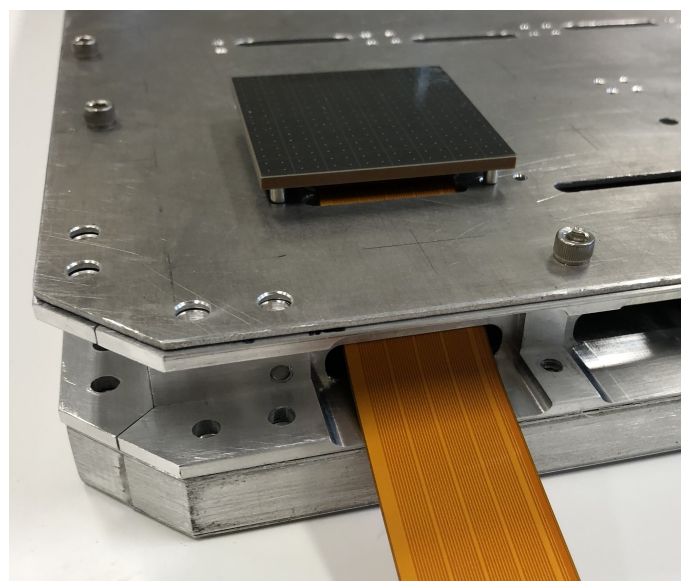

Figure 4: Picture showing the $\mathrm{C}$ channel and focal plane cut outs for routing cables as well as the hole patterns for the SiPM arrays as well as with one SiPM array installed in the assembly. enced during ascent, the honeycomb structure was drilled with holes to vent the cells and the assembly tested in a vacuum chamber.

With the honeycomb as a base, the focal plane can be thin and still stay flat despite the over one thousand holes required to mount the SiPMs, route the cables, and mount support structures. The plate was laser cut out of a $1 \mathrm{~mm}$ thick sheet of aluminum and the holes finished by hand. The plane is supported in the middle by aluminum standoffs and on the edge of the RICH focal plane by $\mathrm{C}$ channels shown in figure 4 . The $\mathrm{C}$ channels include holes to feed cables from the SiPMs distributed across the focal plane to the electronics located near the edges of the assembly. The focal plane and honeycomb are then connected using staggered fasteners around the edges to form a solid piece to keep the enclosure as light-tight as possible. Once assembled the sandwich of the thin focal plane and the honeycomb provides a rigid platform that is low-mass, meets the flatness requirements for the detector, and provides enclosed cable routing for the SIPMs.

\subsection{Radiator Assembly}

A challenge of RICH detectors is characterizing the radiator component index of refraction and determining the particle trajectory through the radiator accurately enough. The aerogel tiles used by HELIX are $10 \mathrm{~cm} \times 10 \mathrm{~cm} \times 1 \mathrm{~cm}$ and are individually supported by black anodized aluminum frames to protect the relatively brittle aerogel from mechanical damage and provide 
fiducial points when measuring the properties of the aerogel. The black anodizing cuts down on stray reflections that occur when a particle passes through the aerogel near the edge of the tile. To assist in calibration, the entire radiator assembly can be lifted off the RICH and placed into a test setup. This design choice is also important so that the aerogel can be shipped separately to avoid damaging the tiles.

Similarly to the RICH focal plane, the radiator assembly is required to be thin but rigid enough to provide a fiducial plane for the detector components. Due to the smaller size of $0.6 \mathrm{~m} \times 0.6 \mathrm{~m}$ the mechanical structure was machined out of a solid piece of aluminum and the aerogel frames held securely to it with M5 screws. To maintain the accuracy of the aerogel characterization the RICH assembly requires that the radiator assembly be located accurately relative to the magnet and the other detectors. The aerogel mount has holes that locate it against the magnet with pins, and provide an accurate relative position to the tracking chamber in the magnet bore.

\section{Conclusions}

The conceptual and primary mechanical designs of the HELIX RICH detector have been completed. The majority of the main mechanical components have been constructed and qualified with mechanical fits and quality control metrology completed. The electronics and cabling have passed two rounds of functionality testing, with a goal to move to production by fall, 2019. Once assembled, the thermal designs of the RICH will be verified before it is integrated with the other subsystems in the instrument gondola. A thermal-vacuum test of the entire payload will then be performed in Spring of 2020 to qualify the instrument for flight later in the year.

\section{References}

[1] S.P. Wakely et al., Prospects for High Energy Light Isotope Measurements on Balloons, in proceedings of the 34th International Cosmic Ray Conference, PoS ( ICRC2015) 682.

[2] J. Beatty et al. Cosmic-ray isotope measurements with HELIX, in proceedings of the 35th International Cosmic Ray Conference, PoS (ICRC2017) 226.

[3] T. Hams, et al., Measurement of the Abundance of Radioactive ${ }^{10}$ Be and Other Light Isotopes in Cosmic Radiation up to $2 \mathrm{GeV}$ Nucleon with the Balloon-borne Instrument ISOMAX Astrophys. J. 611, 892 (2004)

[4] A. Putze et al., Systematic uncertainties on the cosmic-ray transport parameters, 2010, A\&A 516, A66

[5] P. Allison et al., Cosmic-ray isotope measurements with HELIX, in proceedings of the 36th International Cosmic Ray Conference, PoS ( ICRC2019) 121.

[6] M. Tabata et al. Developing a silica aerogel radiator for the HELIX ring-imaging Cherenkov system, NIM A 2019, https://doi.org/10.1016/j.nima.2019.02.006, [arXiv:1901.06663].

[7] M. Tabata et al., Production of Silica Aerogel Radiator Tiles for the HELIX RICH Detector, in proceedings of the 36th International Cosmic Ray Conference, POS ( ICRC2019) 133.

[8] T. Rosin et al. Calibration of the Aerogel Tiles for the HELIX RICH, in proceedings of the 36th International Cosmic Ray Conference, PoS (ICRC2019) 133. 